PROCEEDINGS OF THE

AMERICAN MATHEMATICAL SOCIETY

Volume 133, Number 6, Pages 1763-1768

S 0002-9939(05)07851-2

Article electronically published on January 14, 2005

\title{
ON THE SOLITARY WAVE PULSE IN A CHAIN OF BEADS
}

\author{
J. M. ENGLISH AND R. L. PEGO
}

(Communicated by Mark J. Ablowitz)

\begin{abstract}
We study the shape of solitary wave pulses that propagate in an infinite chain of beads initially in contact with no compression. For this system, the repulsive force between two adjacent beads is proportional to the $p^{\text {th }}$ power of the distance of approach of their centers with $p=\frac{3}{2}$. It is known that solitary wave solutions exist for such a system when $p>1$. We prove extremely fast, double-exponential, asymptotic decay for these wave pulses. An iterative method of solution is also proposed and is seen to work well numerically.
\end{abstract}

\section{INTRODUCTION}

This note examines the properties and shape of pulse solutions of the $2^{\text {nd }}$-order differential-difference equation

$$
r^{\prime \prime}(x)=r^{p}(x-1)-2 r^{p}(x)+r^{p}(x+1), \quad-\infty<x<\infty,
$$

where $p>1$ is a real number.

Motivation for studying equation (1) comes from the work of Coste et al. 3]. These authors studied experimentally the propagation of solitary wave pulses through a long chain of identical steel spheres. In such a chain, the laws of motion for the system state that the acceleration of each ball is proportional to the difference between the compression force of the two neighboring balls. For two adjacent elastic steel spheres with radius $a$, the force between them according to Hertz's law is $F_{c o m p} \propto r^{\frac{3}{2}}$, where $r$ is the distance of approach of their centers.

Thus, the equation of motion for the $n^{\text {th }}$ bead in the chain has the form

$$
\partial_{t}^{2} u_{n}=A\left(r_{n}^{\frac{3}{2}}-r_{n+1}^{\frac{3}{2}}\right)
$$

Here $A$ is a physical constant, $u_{n}$ is the displacement of the $n^{\text {th }}$ ball from static equilibrium, and $r_{n}$ is the distance of approach between centers of the $(n-1)^{\text {st }}$ and $n^{\text {th }}$ balls. In this paper our interest focuses on the case when the chain of spheres is not pre-compressed in equilibrium, so that at rest the balls are just touching with no static force. Then $r_{n}=u_{n-1}-u_{n}$ is simply the compression from equilibrium of two adjacent steel balls, and the wave propagation equation (2) is purely nonlinear.

Received by the editors February 19, 2004.

2000 Mathematics Subject Classification. Primary 35B40, 35C15, 35Q51.

Key words and phrases. Solitons, lattice, differential-difference equations, wave propagation.

This material is based upon work supported by the National Science Foundation under grants DMS 00-72609 and DMS 03-05985.

(C)2005 American Mathematical Society Reverts to public domain 28 years from publication 
It is no trouble to consider the more general homogeneous force law $F_{\text {comp }} \propto r^{p}$, where $p>1$, and we will do so in this paper. For $p=\frac{3}{2}$, MacKay [6] applied the theory of Friesecke and Wattis [5] to prove the existence of a constant-velocity pulse solution $r_{n}=r(n-c t)$ in this one-dimensional chain. Essentially the same argument works also for $p>1$, as shown by Ji and Hong [7. Let $x=n-c t$ and subtract $\partial_{t}^{2} u_{n}$ from $\partial_{t}^{2} u_{n-1}$ to get that $r(x)$ must satisfy

$$
r^{\prime \prime}(x)=\frac{A}{c^{2}}\left[r^{p}(x-1)-2 r^{p}(x)+r^{p}(x+1)\right] .
$$

This equation is equivalent to (1) through a simple scaling. Given a specific solution $\tilde{r}(x)$ of (11), $r=b \tilde{r}$ satisfies (3) provided $b^{p-1}=A / c^{2}$. Accordingly, we will set $A / c^{2}=1$ from this point forward without loss of generality.

The works of Mackay and Ji and Hong yield positive solutions with

$$
\lim _{x \rightarrow \pm \infty} r(x)=0
$$

but this result does not say much about the asymptotic decay of the pulse solutions. In fact, pulse solutions decay extremely fast. We will prove below that pulse tails can be bounded above by a double exponential function of the form $f(x)=k^{p^{x}}$, where $0<k<1$. This is approximately the form of decay obtained in the approximation theory of Chatterjee 2. We will also compute the pulse shape numerically using a simple iterative method and compare its decay to the bound.

\section{INTEGRAL REFORMULATION}

First, we rewrite equation (1) in an equivalent integral form. To do this, we will use the Fourier transform

$$
\hat{r}(k)=\mathcal{F}\{r(x)\} \equiv \frac{1}{2 \pi} \int_{-\infty}^{\infty} r(x) e^{-i k x} \mathrm{~d} x,
$$

and its associated inverse transform

$$
r(x)=\mathcal{F}^{-1}\{\hat{r}(k)\} \equiv \int_{-\infty}^{\infty} \hat{r}(k) e^{i k x} \mathrm{~d} k .
$$

Transforming equation (11) yields

$$
\hat{r}(k)=\left(\frac{\sin \frac{1}{2} k}{\frac{1}{2} k}\right)^{2} \widehat{r^{p}}(k) .
$$

Next we will apply the inverse transform to equation (5), using the identity

$$
\hat{f} \cdot \hat{g}=\frac{1}{2 \pi} \widehat{f * g}
$$

where $(f * g)(x)=\int_{-\infty}^{\infty} f(x-y) g(y) \mathrm{d} y$ is the convolution of $f$ and $g$. We note that

$$
\mathcal{F}^{-1}\left\{\left(\frac{\sin \frac{1}{2} k}{\frac{1}{2} k}\right)^{2}\right\}=2 \pi \Lambda(x)
$$

where the tent function $\Lambda(x)$ is given by the following:

$$
\Lambda(x)=(1-|x|)_{+}= \begin{cases}1-|x|, & -1 \leq x \leq 1 \\ 0, & |x|>1\end{cases}
$$


Applying the inverse transform to equation (5), we get

$$
r(x)=\left(\Lambda * r^{p}\right)(x) .
$$

It is straightforward to check that any continuous solution of this equation is a $C^{2}$ (hence $C^{\infty}$ ) solution of (11). Conversely, any solution of (11) with sufficiently rapid decay must satisfy (6). This applies to the solutions of MacKay and Ji and Hong, since these are continuous and square-integrable on $\mathbb{R}$. The reason is that the theory of [5] implies $r(x)=Q(x+1)-Q(x)$ where $\int_{-\infty}^{\infty} Q^{\prime}(s)^{2} d s<\infty$, and so

$$
\int_{-\infty}^{\infty} r(x)^{2} d x=\int_{-\infty}^{\infty}\left(\int_{0}^{1} Q^{\prime}(x+s) d s\right)^{2} d x \leq \int_{-\infty}^{\infty} \int_{0}^{1} Q^{\prime}(x+s)^{2} d s d x<\infty .
$$

\section{Asymptotic Behavior}

We will exploit equation (6) to show the extremely fast decay of the wave profile. This is done in the following theorem. Of course, the bound that we obtain can be scaled appropriately for all other positive values of $A$ and $c$.

Theorem 1. Assume that $r(x)$ is a positive solution of $r(x)=\left(\Lambda * r^{p}\right)(x)$ such that $\lim _{x \rightarrow \infty} r(x)=0$. Then for any $\varepsilon>0$, if $x_{0} \in \mathbb{R}$ is such that $r(x) \leq$ $\min \left(\varepsilon^{p}, 2^{-1 /(p-1)}\right)$ for all $x \geq x_{0}$, then

$$
r(x) \leq \varepsilon^{p^{x-x_{0}}} \quad \text { for all } x \geq x_{0} .
$$

Proof. Let

$$
F_{x_{0}}=\sup _{x \geq x_{0}} r(x), \quad L_{x_{0}}=\int_{-\infty}^{x_{0}} \Lambda\left(x_{0}-y\right) r^{p}(y) \mathrm{d} y .
$$

We begin by breaking apart at $x_{0}$ the integral on the right-hand side of (6):

$$
r(x)=\int_{-\infty}^{x_{0}} \Lambda(x-y) r^{p}(y) \mathrm{d} y+\int_{x_{0}}^{\infty} \Lambda(x-y) r^{p}(y) \mathrm{d} y .
$$

Since $\Lambda(x)$ is decreasing for positive $x$, we have that $0 \leq x_{0}-y \leq x-y$ implies $\Lambda\left(x_{0}-y\right) \geq \Lambda(x-y)$. Hence for $x \geq x_{0}$,

$$
r(x) \leq \int_{-\infty}^{x_{0}} \Lambda\left(x_{0}-y\right) r^{p}(y) \mathrm{d} y+\left[\sup _{y \geq x_{0}} r^{p}(y)\right] \int_{-\infty}^{\infty} \Lambda(x-y) \mathrm{d} y,
$$

whence $F_{x_{0}} \leq L_{x_{0}}+F_{x_{0}}^{p}$. Next, we bound $L_{x_{0}+1}$ in terms of $F_{x_{0}}$ :

$$
L_{x_{0}+1}=\int_{x_{0}}^{x_{0}+1} \Lambda\left(x_{0}+1-y\right) r^{p}(y) \mathrm{d} y \leq \sup _{y \geq x_{0}} r^{p}(y) \cdot \int_{0}^{1} \Lambda(1-y) \mathrm{d} y=\frac{1}{2} F_{x_{0}}^{p} .
$$

Now observe that $F_{x_{0}+1} \leq F_{x_{0}}$, and hence if $x_{0} \in \mathbb{R}$ is sufficiently large so that $\sup _{x \geq x_{0}} r(x)^{p-1} \leq \frac{1}{2}$,

$$
F_{x_{0}+1} \leq L_{x_{0}+1}+F_{x_{0}+1} F_{x_{0}}^{p-1} \leq L_{x_{0}+1}+\frac{1}{2} F_{x_{0}+1},
$$

whence $F_{x_{0}+1} \leq F_{x_{0}}^{p}$. Recursively it follows that $F_{x_{0}+n} \leq F_{x_{0}}^{p^{n}}$ for $n=1,2, \ldots$

To finish the proof, for any $x \geq x_{0}$ write $x=x_{0}+n+\Delta x$ where $0 \leq \Delta x<1$. Then

$$
r(x) \leq F_{x_{0}+n} \leq F_{x_{0}}^{p^{x-x_{0}-\Delta x}} \leq F_{x_{0}}^{p^{x-x_{0}-1}} .
$$

Given $\varepsilon \in(0,1)$ we may pick $x_{0}$ so large that $F_{x_{0}}^{1 / p} \leq \varepsilon$. Then (7) follows. 


\section{ITERATIVE METHOD FOR COMPUTATION}

We consider a simple iterative method for solving equation (6). Starting with $\phi_{0}(x)=\Lambda(x)$, for $m=1,2, \ldots$ compute successively

$$
\begin{aligned}
\tilde{\phi}_{m}(x) & =\left(\Lambda * \phi_{m-1}^{p}\right)(x), \\
C_{m} & =\left(\int_{-\infty}^{\infty} \phi_{m-1}(x) \mathrm{d} x\right)\left(\int_{-\infty}^{\infty} \tilde{\phi}_{m}(x) \mathrm{d} x\right)^{-1}, \\
\phi_{m}(x) & =C_{m} \tilde{\phi}_{m}(x) .
\end{aligned}
$$

The normalization of the iterates implies $\int_{-\infty}^{\infty} \phi_{m}(x) d x=1$ for all $m$. Moreover, integration yields $\int_{-\infty}^{\infty} \tilde{\phi}_{m}(x) d x=\int_{-\infty}^{\infty} \phi_{m-1}(y)^{p} d y$, and then the fact that $0 \leq$ $\Lambda(x-y) \leq 1$ yields the pointwise bound

$$
\phi_{m}(x)=\int_{-\infty}^{\infty} \Lambda(x-y) \phi_{m-1}(y)^{p} d y\left(\int_{-\infty}^{\infty} \phi_{m-1}(y)^{p} d y\right)^{-1} \leq 1 .
$$

Despite these bounds, the existence of $\lim _{m \rightarrow \infty} \phi_{m}$ remains an open question and apparently a delicate one. Suppose, however, that $\phi_{*}(x)=\lim _{m \rightarrow \infty} \phi_{m}(x)$ exists for all $x$ uniformly, with $\phi_{*}(x)>0$ and $\int_{-\infty}^{\infty} \phi_{*}(x) d x=1$. If this is the case, then

$$
\tilde{\phi}_{*}(x)=\lim _{m \rightarrow \infty}\left(\Lambda * \phi_{m}^{p}\right)(x)
$$

exists, and

$$
C_{m} \rightarrow C_{*}=\left(\int_{-\infty}^{\infty} \phi_{*}(x) \mathrm{d} x\right)\left(\int_{-\infty}^{\infty} \tilde{\phi}_{*}(x) \mathrm{d} x\right)^{-1} .
$$

Thus, $\phi_{*}(x)=C_{*}\left(\Lambda * \phi_{*}^{p}\right)(x)$, and through scaling, $C_{*}^{1 /(p-1)} \phi_{*}(x)$ is a solution of equation (6).

Upon discretization, this method appears to converge well in practice. We have used the method to compute a limiting shape that solves a discrete version of equation (6). Figure 1 shows the limit of the iterative method multiplied by $C_{*}^{1 /(1-p)}$ for $p=\frac{3}{2}$. Figure 2 shows a logarithmic plot of the same function along with an upper bound given by Theorem 1. These figures display the $100^{\text {th }}$ iteration of the method, implemented on a lattice with spacing $\Delta x=1 / 100$.

As one can see in these plots, the limiting solution appears to both exist and be decreasing for $x>0$. In fact, Benjamin et al. show in [1] that convolution operators of the type our method uses preserve the decreasing property of $\phi_{0}(x)$. So, there is strong evidence that there exists a solitary pulse solution to equation (6) that is decreasing on $(0, \infty)$. This leads us to an improved (though not proven) estimate of the asymptotic decay of the solitary pulse solution. This is offered in the following final result:

Theorem 2. Assume that $r(x)$ is a positive solution of $r(x)=\left(\Lambda * r^{p}\right)(x)$ such that $\lim _{x \rightarrow \infty} r(x)=0$. Also assume that $r(x)$ is decreasing for $x>0$. Then for any $x_{0}>0$ such that $r\left(x_{0}\right)<1$, for all $x \geq x_{0}$ we have

$$
r(x) \leq r\left(x_{0}\right)^{p^{x-x_{0}-1}} .
$$

Furthermore, if $x-x_{0}$ is a positive integer, then

$$
r(x) \leq r\left(x_{0}\right)^{p^{x-x_{0}}} .
$$




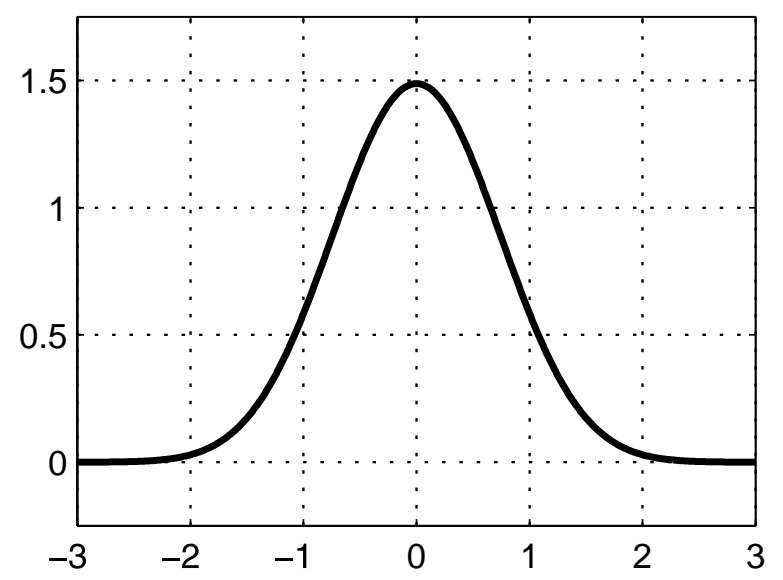

Figure 1. A plot of $C_{*}^{\frac{1}{p-1}} \phi_{*}(x) \approx r(x)$ (solid line) as computed by the proposed iterative scheme.

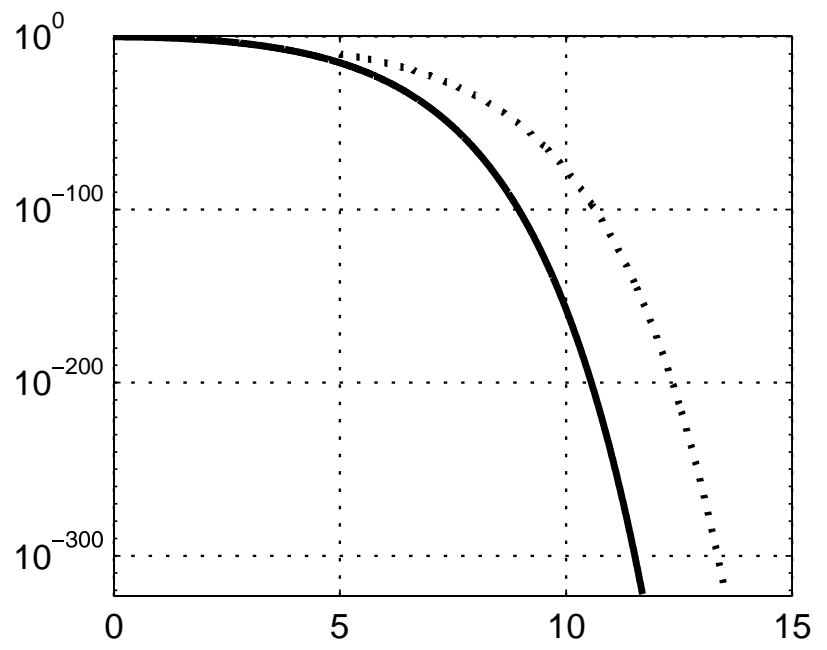

FiguRE 2. A semi-logarithmic plot of $C_{*}^{\frac{1}{p-1}} \phi_{*}(x) \approx r(x)$ (solid line) and the upper bound on $r(x)$ given by equation (8) for $x_{0}=5$ (dotted line). Here $p=\frac{3}{2}$.

Proof. Suppose $x_{0}>0$ and $r\left(x_{0}\right)<1$. Because $r(x)$ is decreasing for positive $x$, $\sup _{y \in[-1,1]} r^{p}(x+1-y)=r^{p}(x)$. So, for all $x \geq x_{0}$,

$$
r(x+1)=\int_{-1}^{1} \Lambda(y) r^{p}(x+1-y) \mathrm{d} y \leq \sup _{y \in[-1,1]} r^{p}(x+1-y) \int_{-1}^{1} \Lambda(y) \mathrm{d} y=r^{p}(x) .
$$


This implies that for any positive integer $n, r\left(x_{0}+n\right) \leq r\left(x_{0}\right)^{p^{n}}$. Now, pick $\Delta x \in[0,1)$ and let $x=x_{0}+n+\Delta x$. Then

$$
r(x)=r\left(x_{0}+n+\Delta x\right) \leq r\left(x_{0}+n\right) \leq r\left(x_{0}\right)^{p^{n}}=r\left(x_{0}\right)^{p^{x-\left(x_{0}+\Delta x\right)},}
$$

and the result follows.

A simple consequence of Theorem 2 is that $\lim _{x \rightarrow \infty} r(x+1) / r(x)=0$.

\section{REFERENCES}

[1] T. B. Benjamin, J. L. Bona and D. K. Bose, Solitary-wave solutions of nonlinear problems, Philos. Trans. Roy. Soc. London Ser. A 331 (1990) 195-244. MR1062564 (91h:35309)

[2] A. Chatterjee, Asymptotic solution for solitary waves in a chain of elastic spheres, Phys. Rev. E 59 (1998) 5912-5918.

[3] C. Coste, E. Falcon, S. Fauve, Solitary waves in a chain of beads under Hertz contact, Phys. Rev. E 56 (1997) 6104-6117.

[4] G. Friesecke, R. L. Pego, Solitary waves on FPU lattices: I. Qualitative properties, renormalization and continuum limit, Nonlinearity 12 (1999) 1601-1627. MR1726667(2001h:37158)

[5] G. Friesecke, J. A. D. Wattis, Existence theorem for solitary waves on lattices, Commun. Math. Phys. 161 (1994) 391-418. MR1266490 (95c:35205)

[6] R. S. MacKay, Solitary waves in a chain of beads under Hertz contact, Phys. Lett. A 251 (1999) 191-192.

[7] J.-Y. Ji, J. Hong, Existence criterion of solitary waves in a chain of grains, Phys. Lett. A 260 (1999) 60-61. MR,1714633 (2000f:82019)

Department of Mathematics, University of Wisconsin, Madison, Wisconsin 53705

E-mail address: english@math.wisc.edu

Department of Mathematics and Institute for Physical Sciences and Technology, University of Maryland, College Park, Maryland 20742

E-mail address: rlp@math.umd.edu

Current address: Department of Mathematical Sciences, Carnegie Mellon University, Pittsburgh, Pennsylvania 15213

E-mail address: rpego@cmu.edu 